\title{
Determination of Size-Dependent Energy Bandgap of Germanium (Ge) nanostructure
}

\author{
Adem Beriso \\ Madda Walabu University, College of Natural and Computational Science, Department of Physics, Bale-Robe, \\ Ethiopia; P.box: 247
}

\begin{abstract}
Energy band gap is fundamentally important for the properties of a solid. Most of a material's behaviors, such as conductivity, optical transitions, or electronic transitions, depend on it. Any change of the energy band gap may significantly alter the material's physical and chemical properties. Change of the energy band gap occurs when the size of a solid is reduced to the nanometer length scale. Germanium is a semiconductor element and it has indirect energy band gap when it is in its bulk structure. Its energy band gap changes from indirect gap to direct gap after its size reduced to the nanometer scale which is responsible to many novel properties of the material. In this research paper, I investigated dependence of energy band gap of germanium nanostructure (the case of quantum dot) on the size of the dot and the findings are in agreement with other theoretical and research results.
\end{abstract}

Keywords: Energy gap; novel properties; size; nanometer scale; quantum dot

DOI: $10.7176 /$ APTA/77-01

Publication date:May $31^{\text {st }} 2019$

\section{Introduction}

Semiconductor nanomaterials have been attracted considerable attention for the last decades due to their exceptional physical and chemical properties, which make them as the best advanced functional materials for nanodevices $[1,2]$. As a result of the energy band gap of semiconductor materials playing a fundamental role in electrical and optical properties, it is important and necessary to study on the band-gap change in order to gain a better understanding for their relevant properties [3]. Over the last few decades, it was well established that below a particular size (in the nanoscale region which extends from $1 \mathrm{~nm}$ to $100 \mathrm{~nm}$ ), physico-chemical properties of semiconducting materials become size dependent [4]. This phenomenon attracted wider attention to the integration of optically and electrically active semiconducting materials after nanostructured silicon as well as germanium were observed to show strong photoluminescence, although their bulk counterparts are indirect energy band gap semiconducting material and hence non-radiative. This feature could allow the synthesis of more optically as well as electrically active materials [5].

The size dependent physical, chemical, electrical, optical and even biological properties of nanomaterials arise from effects, such as quantum confinement or surface plasmon resonance. Thus, these particles are highly promising for many optical, electronic, diagnostic, chemical, or biomedical applications, because the particle properties can be tuned to optimize the physical and chemical performance. Detailed knowledge of size, shape, molecular weight, and composition of these nanoparticles is required to understand their physical and chemical properties $[6,7]$.

\section{Quantum confinement effect}

When one or more dimensions of a particle are brought to the nanoscale region, the quantum confinement effect sets in [8]. This concept can be explained using the term band gap, a property that is characteristic of semiconductors. The band gap can be defined as the difference in energy between the valence and conduction band and is typically in the range of $1-3 \mathrm{eV}$ for semiconductors [9]. In bulk materials, the energy bands are a continuum of energy levels arising from the overlapping of the atomic orbital's due to the large number of molecules. When a semiconductor is brought to the nanoscale, containing lesser number of molecules, the quantized energy levels become distinct. Thus, the addition or removal of atoms significantly changes the energy levels within the band making the band gap size dependent [10]. 
Energy levels in bulk semiconductors

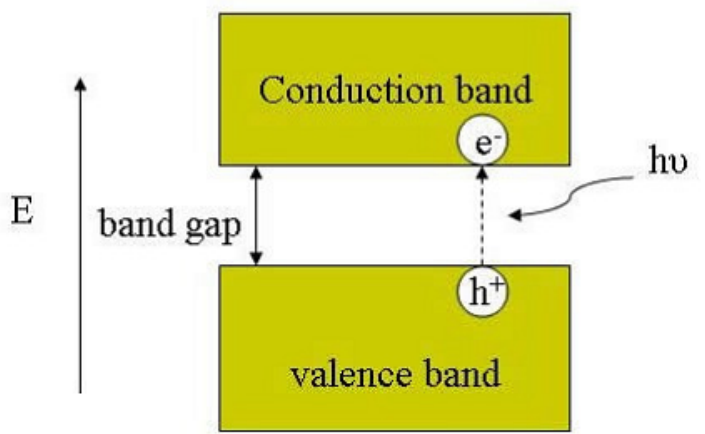

Energy levels in a quantum dot

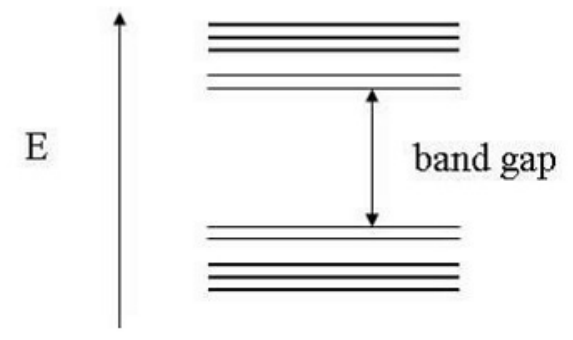

Figure 1: A Schematic diagram of the energy levels in a bulk semiconductor and a nanocrystal [11].

The figure above illustrates the excitation of an electron into the conduction band thus leaving a hole in the valence band. An electron-hole pair is called an exciton, and the natural physical separation between them is called the excitonic Bohr radius and is characteristic of each material. Thus when a semiconducting material approaches a size nearing its Bohr excitonic radius (nanoscale), the exciton is said to be confined within the particle and is called a quantum dot [11].

\section{Formulation of quantum confinement model (QCM)}

This model is based on the electronic confinement in dot like structure of semiconductor ( $\mathrm{Ge}, \mathrm{Si}$, etc). This model is based on the effective mass approximation theory. In this model, the luminescence process is attributed to an energy shift of carriers (electrons and holes) and is proportional to $L^{-2}, L$ being diameter of nanostructure(here Ge nanostructure quantum dot is considered). Luminescence is light that usually occurs at low temperature and thus form a cold body radiation [12]. Quantum dot (QD) nanostructure is modeled by a sphere of diameter $L$, so that its HOMO-LUMO energy gap relation is given as

$$
E_{\mathrm{g}}{ }^{n}=E_{\mathrm{g}}{ }^{b}+\frac{V_{1}}{L_{1} a_{1}}
$$

Quantum wire (nanowire) can be modeled as a cylinder with HOMO-LUMO energy gap,

$$
E_{\mathrm{g}}{ }^{n}=E_{\mathrm{g}}{ }^{b}+\frac{V_{2}}{L_{2} a_{2}}
$$

Quantum well (QW) nanostructure is modeled as hemisphere so that its HOMO-LUMO energy is parabola and its energy relation is given as

$$
E_{\mathrm{g}}{ }^{n}=E_{\mathrm{g}}{ }^{b}+\frac{V_{3}}{L_{3}{ }^{a_{3}}}
$$

where $E_{\mathrm{g}}{ }^{b}$ and $E_{\mathrm{g}}{ }^{n}$ are the energy gaps of the bulk and confined structures respectively for the given geometries mentioned above; $L_{1}, L_{2}$ and $L_{3}$ are the diameters of the sphere, the cylinder and the hemisphere respectively and $V_{1}, V_{2}$ and $V_{3}$ and $a_{1}, a_{2}$ and $a_{3}$ are constants that can be found by empirical fit. $a$ (for $a_{1}, a_{2}, a_{3}$ ) is the porosity level and ranges from 1.25 to 1.85 and $V$ (for $V_{1}, V_{2}, V_{3}$ ) is the value for the passivation of atoms/ions on germanium nanostructure. In this work $0.67 \mathrm{eV}$ will be used for energy band gap of bulk germanium semiconductor in equation (1) above in the following section.

\section{Results and Discussions}

In quantum confinement model discussed above energy gap of nanomaterials is mathematically expressed in terms of energy gap of their counterpart bulk materials, their diameter and the constants $V$ and $a$. Here germanium nanostructure (Ge-ns) as quantum dot nanostructure is considered and the dependence of its energy gap $\left(E_{\mathrm{g}}{ }^{n}\right)$ on its size and on the constants $V$ and $a$ will be investigated. These dependence can be generated from equation (1) above by applying Matlab program.

If $\mathrm{v}=4.5$ for $\mathrm{a}=1.32$ and 1.42 with $E_{\mathrm{g}}{ }^{b}=0.67 \mathrm{eV}$ (energy gap of bulk germanium) and d which varies from $1 \mathrm{~nm}$ to $10 \mathrm{~nm}$ are used in eq.(1) figure 2 below is generated. As it can be seen from this figure energy gap of Ge nanodot $\left(E_{\mathrm{g}}{ }^{n}\right)$ varies with the diameter of the dot. It increases as size of the dot decreases. 


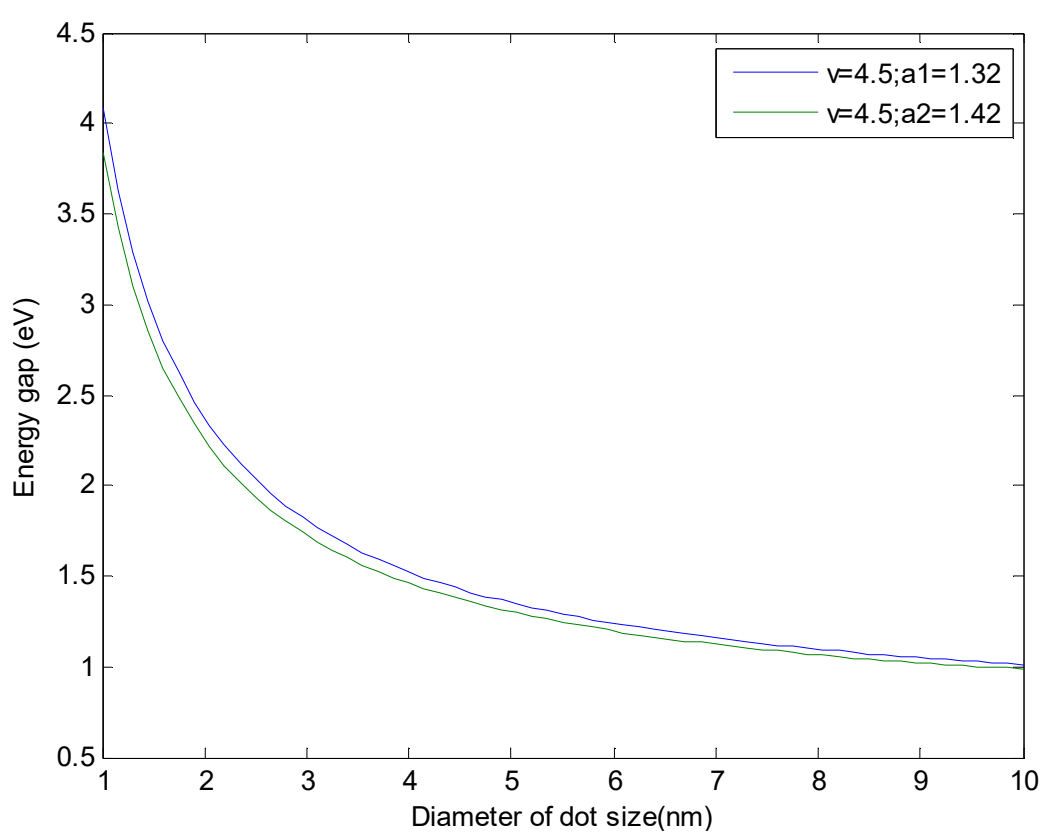

Figure 2: Variation of energy gap of Ge nanostructure (quantum dot) with the diameter of the quantum dot. The upper line (blue) is for $\mathrm{a} 1=1.32$ and the lower line (green) is for $\mathrm{a} 2=1.42$. For both lines $\mathrm{v}=4.5$.

If $\mathrm{v}=6.2$ for $\mathrm{a}=1.32$ and 1.42 with $E_{\mathrm{g}}{ }^{b}=0.67 \mathrm{eV}$ and $\mathrm{d}$ which varies from $1 \mathrm{~nm}$ to $10 \mathrm{~nm}$ are used in eq.(1) figure 3 below is obtained. As we observe from this figure energy gap of Ge nanodot $\left(E_{\mathrm{g}}{ }^{n}\right)$ varies with the diameter of the dot. In the same fashion as figure 2 above it increases as size of the dot decreases. Comparing figures 2 and 3 , we see that for smaller value of a $(\mathrm{a}=1.32)$ energy gap is large (blue line) but for larger value of $\mathrm{a}(\mathrm{a}=1.42)$ energy gap is small (green line). This implies that as we increase porosity level (a stands for porosity level) the value of energy gap of Ge nanostructure quantum dot decreases. Again, from these two figures we see that around $\mathrm{d}=10 \mathrm{~nm}$ energy gap has smaller value when $\mathrm{v}=6.2$ (figure 3 ) than when $\mathrm{v}=4.5$ (figure 2 ) but around $\mathrm{d}=1 \mathrm{~nm}$ energy gap has larger value when $v=6.2$ (figure 3 ) than when $v=4.5$ (figure 2). This implies that energy gap increases more rapidly as we increase the value of passivation, $\mathrm{v}$.

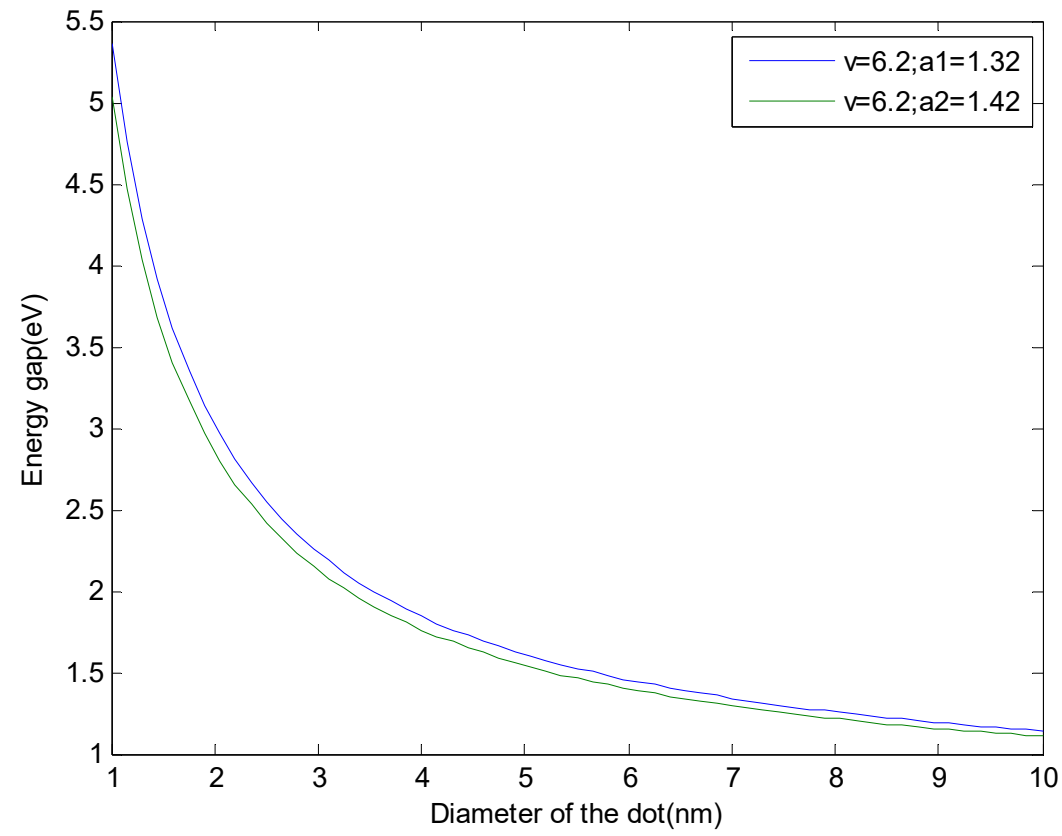

Figure 3: Variation of energy gap of Ge nanostructure (quantum dot) with the diameter of the quantum dot. The upper line (blue) is for $\mathrm{a} 1=1.32$ and the lower line (green) is for $\mathrm{a} 2=1.42$. For both lines $\mathrm{v}=6.2$.

For the same values of $\mathrm{v}$ and a's the energy gap of germanium quantum dot is less than that of silicon quantum dot around $1 \mathrm{~nm}$ diameter of the dots from my previous work. This is because the energy gap of bulk germanium $(0.67 \mathrm{eV})$ is less than the energy gap of bulk silicon $(1.12 \mathrm{eV})$.

Figures 4 and 5 are obtained in the same way as figures 2 and 3 but here we used the porosity level from its 
minimum value (1.25) to maximum value (1.85) by adjusting the intervals with a value 0.20 . In both figures 4 and 5 the same conclusion can be drawn as figures 2 and 3 but the differences we observe between figures 2 and 3 and figures 4 and 5 is that the energy gap difference between energy gaps in figures 4 and 5 is larger than that of figures 2 and 3. This is because the difference between passivation in figures 4 and 5 is larger than that of figures 2 and 3.

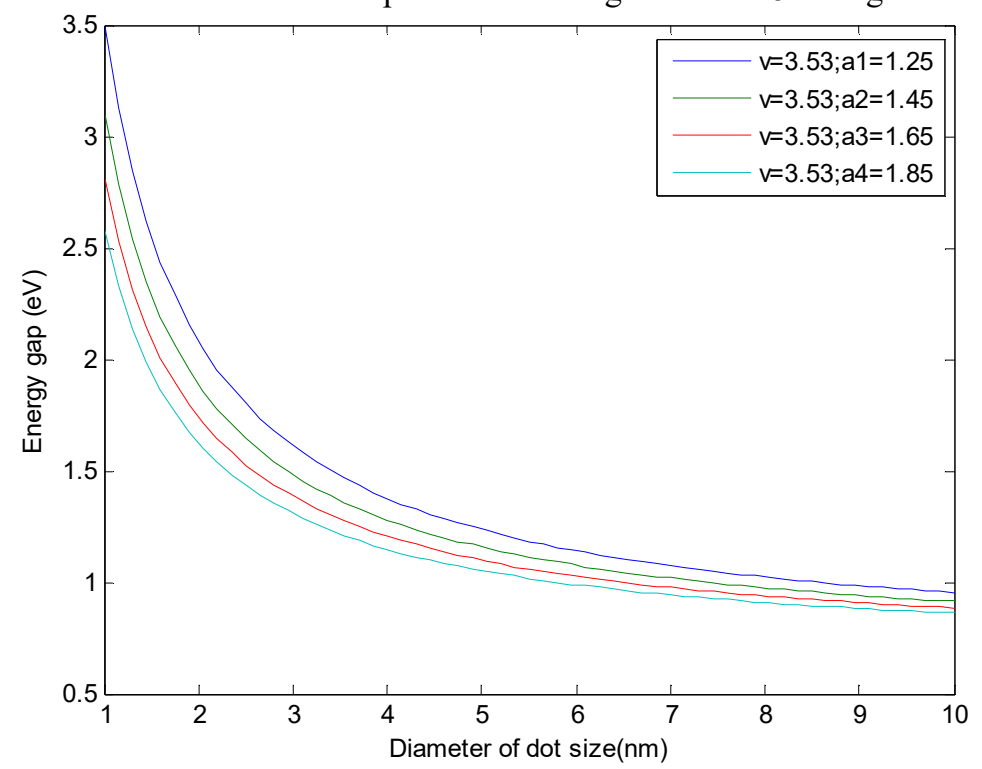

Figure 4: Variation of energy gap of Ge nanostructure (quantum dot) with the diameter of the quantum dot. The different four lines are drown for $\mathrm{a} 1=1.25, \mathrm{a} 2=1.45, \mathrm{a} 3=1.65$ and $\mathrm{a} 4=1.85$. For both lines $\mathrm{v}=3.53$.

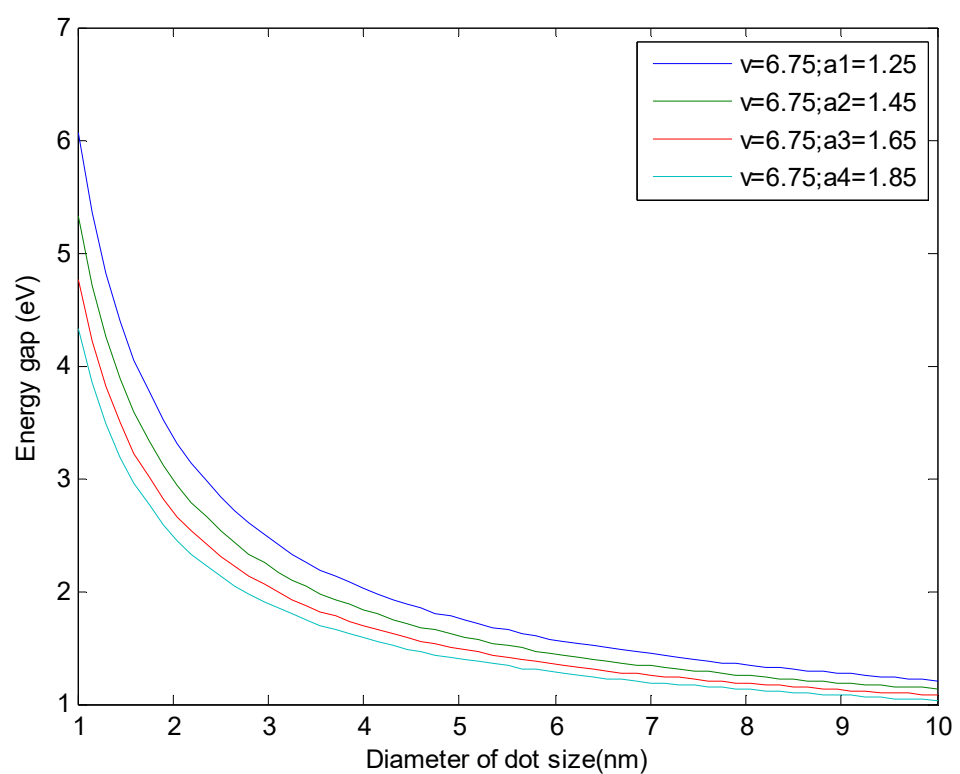

Figure 5: Variation of energy gap of Ge nanostructure (quantum dot) with the diameter of the quantum dot. The different four lines are drown for $\mathrm{a} 1=1.25, \mathrm{a} 2=1.45, \mathrm{a} 3=1.65$ and $\mathrm{a} 4=1.85$. For both lines $\mathrm{v}=6.75$.

\section{Conclusion}

The main objective of this work was to investigate the size dependence of energy band gap of germanium nanostructure (the case of quantum dot). It was shown that energy band gap of germanium quantum dot strongly depends on the size of the dot when the material is reduced to the nanometer range. Here it was observed that as the diameter of the dot decreases the energy gap of the material increases. From this work it was also observed that as the passivation, $\mathrm{v}$ increases (changing $\mathrm{v}$ from some smaller value to some other larger value) energy gap correspondingly increases. In other words, the rate of energy gap increase is more rapid for larger value of $\mathrm{v}$ than for smaller value of v. Finally, it was observed that energy gap is larger for smaller value of porosity level (the a1, a2, a3, a4) than for the larger value of porosity level, i.e., energy gap of germanium quantum dot is inversely proportional to the porosity level of the material. 


\section{References}

1. Klaus Sattler (2002). The energy gap of clusters nanoparticles, and quantum dots, chapter 2.

2. S. Neeleshwar, C. L. Chen, C. B. Tsai, Y. Y. Chen, C. C. Chen, S. G. Shyu and M. S. Seehra (2005). Sizedependent properties of CdSe quantum dots: Physical Review B71, 201307(R).

3. Y. Wang, G. Ouyang, L.L. Wang, L.M. Tang, D.S. Tang and Chang Q. Sun(2008). Size- and compositioninduced band-gap change of nanostructured compound of II-VI semiconductors, Chemical Physics Letters 463, 383-386.

4. Margherita Marsili, Silvana Botti, Maurizia Palummo, Elena Degoli, Olivia Pulci, Hans-Christian Weissker, Miguel A. L. Marques, Stefano Ossicini and Rodolfo Del Sole (2013). Ab Initio Electronic Gaps of Ge Nanodots: The Role of Self-Energy Effects, Journal of Physical Chemistry C, 117, 14229-14234.

5. P. Boroojerdian (2013). Structural and Optical Study of SnO Nanoparticles Synthesized Using MicrowaveAssisted Hydrothermal Route, Int. J. Nanosci. Nanotechnol.,9(2), 95-100.

6. Engin Karabudak, Emre Brookes, Vladimir Lesnyak, Nikolai Gaponik, Alexander Eychmüller, Johannes Walter, Doris Segets, Wolfgang Peukert, Wendel Wohlleben, Borries Demeler and Helmut Cölfen (2016). Simultaneous Identification of Spectral Properties and Sizes of Multiple Particles in Solution with Subnanometer Resolution, Angewandte Chemie International Edition; 55 (39), - S, 11770-11774.

7. Aristides D.Zdetsis (2006). Optical and electronic properties of small size semiconductor nanocrystals and nanoclusters, Rev.Adv.Mater.Sci.11, 56-78.

8. Alexander Konchenko, Yasuo Nakayama, Iwao Matsuda, Shuji Hasegawa, Yoshiaki Nakamura and MasakazuIchikawa (2006). Quantum confinement observed in Ge nanodots on an oxidized Si surface, Physical Review B73, 113311.

9. P.K. Giri, R. Kesavamoorthy, B.K. Panigrahi and K.G.M. Nair (2005). Evidence for fast decay dynamics of the photoluminescence from Ge nanocrystals embedded in $\mathrm{SiO}_{2}$, Solid State Communications 133, $229-234$.

10. C. A.Stephenson, W. A.O’Brien, M. W.Penninger, W. F.Schneider, M.Gillett-Kunnath, J.Zajicek, K. M.Yu, R.Kudrawiec, R. A.Stillwell, and M. A.Wistey (2016). Band structure of germanium carbides for direct bandgap silicon photonics, Journal of Applied Physics120, 053102.

11. Sujay Prabakar (2010). Synthesis and Characterization of Silicon and Germanium Nanocrystals and Titanium Disulphide Nanostructures, Victoria University of Wellington.

12. S. Mukherjee, A. Pradhan, T. Maitra, S. Mukherjee, A. Nayak, S. Bhunia (2017). Phase selective growth of Ge Nanocrystalline films by ionized cluster beam deposition technique and photo-oxidation study, Advanced Materials Letters, 8(9), 891-896. 